\title{
Mutation de l'examen de fin de collège en Afrique subsaharienne
}

Evolution of the examination at the end of college in sub-Saharan Africa

Cambio del examen existente a la finalización del ciclo básico secundario en

África subsahariana

Jean-Marc Bernard et François Robert

\section{OpenEdition}

Édition électronique

URL : https://journals.openedition.org/ries/1392

DOI : 10.4000/ries. 1392

ISSN : 2261-4265

Éditeur

France Education international

Édition imprimée

Date de publication : 1 décembre 2004

Pagination : 53-60

ISBN : 978-2-85420-564-0

ISSN : $1254-4590$

Référence électronique

Jean-Marc Bernard et François Robert, « Mutation de l'examen de fin de collège en Afrique subsaharienne », Revue internationale d'éducation de Sèvres [En ligne], 37 | décembre 2004, mis en ligne le 18 novembre 2011, consulté le 05 juillet 2021. URL : http://journals.openedition.org/ries/1392 ; DOI : https://doi.org/10.4000/ries.1392 


\section{Mutation de l'examen de fin de collège en Afrique subsaharienne}

\section{Jean-Marc Bernard François Robert}

L'examen est un reflet fidèle du système scolaire dans lequel il prend place. Il porte en effet la trace des habitudes et des finalités du système, il est chargé d'en dire la réussite ou les carences et surtout, il est la plus précieuse frontière entre l'école et le monde social, en ce qu'il distingue les lauréats des malchanceux, et en ce qu'il détermine souvent la poursuite des études.

Il n'est donc pas étonnant que les pays en développement d'Afrique subsaharienne, qui connaissent enfin aujourd'hui des progrès sensibles dans la fréquentation de leurs écoles, offrent quant à leurs examens plus d'interrogations que de certitudes. L'expansion des scolarités a des effets de structure, elle entraîne une mutation importante du rôle de l'École et ce phénomène prend un tour singulier pour ce qui concerne le premier cycle secondaire, l'école moyenne.

Quand tout milite pour que l'école moyenne devienne, pour de nombreux enfants, le cycle terminal d'une scolarité de base d'une dizaine d'années, l'examen qui en représente le terme revient chargé de missions sociales qui lui sont nouvelles, qu'il n'est pas facile d'assumer, et qui imposent parfois, comme en Mauritanie, des efforts d'imagination pour dessiner une organisation nouvelle.

\section{UN POINT DE RENCONTRE DES TENSIONS}

\section{Petite histoire vécue en Assaba}

Tamchekett, bourgade modérément active de cinq mille âmes, se situe au beau milieu de l'Assaba, province centrale de la Mauritanie, à plus de quatre cents kilomètres à l'est de Nouakchott, dans une zone austère où subsiste un peu d'activité agro-pastorale traditionnelle, associée à un commerce timide. Tamchekett fut, au temps éphémère de l'occupation coloniale, quelque chose comme l'embryon d'une capitale de région, et il en subsiste quelques bâtiments aujourd'hui dégradés, mais Kiffa, voisine de cent kilomètres, l'a détrônée dans ce rôle. Pour peu enviable qu'elle soit, la situation de Tamchekett n'a rien d'exceptionnel en Afrique sahélienne. 
En 2001, le directeur du collège de Tamchekett obtient de son ministère à Nouakchott le droit (concédé à mi-voix) d'ouvrir une classe de seconde, c'est à dire de lycée, pour accueillir les jeunes lauréats locaux du brevet du premier cycle secondaire (ils sont une quarantaine), auxquels aucune autre solution ne peut être proposée. Simplement, cette classe devra fonctionner sans moyens additionnels par rapport à ceux alloués au collège. Disons que cette discrète ouverture d'un lycée s'est opérée sinon par distraction, du moins par défaut d'une gestion réfléchie et anticipée des flux. Disons encore que cette demi-décision est sans doute l'une des pires que l'on pouvait souhaiter pour le système éducatif mauritanien - du moins si elle devait être multipliée par le nombre de bourgades que compte le pays.

En effet, soit les moyens n'arriveront jamais vers ce lycée, et les conditions n'y mèneront à aucun apprentissage sérieux, soit ils y arrivent, mais alors au détriment d'autres emplois, comme le développement des écoles primaires, dont la dernière classe n'est encore fréquentée que par à peine plus d'un enfant sur deux de ce pays. Dans chacun des deux cas d'ailleurs, il faut encore craindre que Tamchekett n'ait que faire de bacheliers férus en culture classique (histoire et poésie), ne seraient-ils qu'une vingtaine par an, et que par conséquent l'opération n'ait produit finalement que des frustrations.

Le collège-lycée de Tamchekett offre une image complète des enjeux qui pèsent sur le développement de l'école moyenne en Afrique subsaharienne. Au cœur de cette figure, nous trouvons l'examen sanctionnant la fin du collège et commandant l'accès aux parcours scolaires supérieurs, examen chargé aujourd'hui de toutes les contradictions des systèmes en expansion.

\section{Un dilemme}

En termes de flux, la situation de l'école moyenne en Afrique sub-saharienne est aujourd'hui à la fois heureuse et malheureuse. C'est une situation de transition dont nul ne voit hélas encore le terme.

Les engagements en faveur de l'éducation pour tous pris à plusieurs reprises, à Jomtien (1990), puis à Dakar (2000) et à des dates diverses par les États semblent enfin, dans de nombreux cas, porter des fruits : l'accès à l'école primaire et l'achèvement du cycle se développent, même dans les pays les moins scolarisés. Des enfants de douze ans environ se présentent donc aujourd'hui en nombre bien plus considérable que dans les années quatre-vingt-dix à la porte de l'enseignement secondaire. La difficulté tient à l'étroitesse des capacités d'accueil de l'enseignement secondaire, voué jusque-là à la formation d'une élite confidentielle et qui voit en quelques années ses effectifs augmenter dans des proportions bien supérieures à celles que connaît l'école primaire! Même dans les situations où l'essentiel des politiques scolaires est accaparé par l'objectif d'achèvement universel du cycle primaire, les plans stratégiques en cours prennent acte de l'augmentation massive des flux qui en résultera pour 
l'école moyenne et proposent des mesures d'accompagnement. C'est le cas des plans décennaux de la Mauritanie, qui anticipent une augmentation des entrées au collège de $60 \%$, et du Sénégal, qui prévoit de doubler les capacités d'accueil au même niveau. C'est le cas du Togo, où, sur la dernière décennie, les effectifs du collège ont été multipliés par 2,3, tandis que ceux de l'école primaire ne l'étaient que par 1,5, et même du Niger, pays pourtant très peu scolarisé, où ces accroissements pour la décennie écoulée sont respectivement de 7,8 et $4,4^{1}$.

Or tous les pays d'Afrique subsaharienne sont dans une situation de rareté des ressources et donc dans l'obligation de les affecter prioritairement à l'école primaire tant que l'expansion de celle-ci n'est pas arrivée à son point d'achèvement. Il faut ajouter à cela que, si chacun s'accorde pour dire que l'école primaire est le moteur du développement économique et social, la chose est beaucoup plus incertaine quand il s'agit du secondaire. Donc, si les États acceptent encore de garder jusqu'à la fin du collège les enfants qui y sont entrés, ils sont contraints, en revanche, de ne réserver l'accès au lycée qu'à une proportion bien plus faible des jeunes au delà de seize ans. Faire l'inverse consisterait à priver l'école primaire de ressources indispensables à son développement, ce serait une injustice importante. On voit bien dans ce contexte quelle position va occuper l'examen qui clôt le premier cycle de l'enseignement secondaire.

\section{Efficacité, équité, justice...}

Cet examen de fin de collège est forcément, dans ce contexte, le lieu de rencontres et de contraintes inconciliables, le lieu d'alchimie d'une justice scolaire qui sera toujours discutable. L'impératif d'efficacité dans la gestion des flux imposerait à cet examen d'être malthusien dans ses résultats. S'il l'était en déclarant admis environ le cinquième des candidats, il opèrerait alors la plus équitable distribution possible pour les enfants du pays entre les ressources affectées à leur éducation et celles consacrées à leur développement. En effet, aucune croissance indue des effectifs de lycée ne viendrait détourner l'argent de sa destination prioritaire, à savoir l'école primaire, dont de trop nombreux enfants sont encore privés. Dans le même temps, l'examen serait le lieu d'une probable injustice envers les adolescents qui, arrivés au terme de dix ans d'école avec des acquis acceptables, se verraient renvoyés à leur foyer sans aucun titre attestant de leur niveau effectif, sans rien qui les distingue de leurs camarades à la formation bien plus fragile et qui ont interrompu plus précocement le cours de leurs études. Au delà même de cette injustice faite à l'individu, c'est l'inefficacité du système qui est à redouter. Non seulement on mesurera alors un rendement interne faible mais encore on aura installé un puissant mécanisme de découragement, propre à ne pas susciter beaucoup d'envie d'école dans les populations.

1. V. Bernard (J.-M.) et Robert (F.), «Quel modèle pour l'école moyenne africaine dans les années à venir? ", Banque Mondiale, communication à la $1^{\text {re }}$ conférence SEIA, Kampala, juin 2003, en ligne sur le site de la Banque Mondiale, rubrique Secondary Education in Africa. 
Cet examen est bien le point focal de toutes ces contradictions. C'est lui qui éclairera de façon très crue le fait que le développement des scolarités, même s'il est d'abord souhaité pour le primaire, s'opère aussi dans le même temps, par progression mécanique, vers les niveaux plus élevés, jusqu'à un certain niveau où, en raison de la rareté des ressources, il sera jugé préférable d'y mettre un frein. Or c'est précisément à l'examen et à aucun autre dispositif qu'échoit ce triste rôle de régulation. Dans la réalité d'ailleurs, on peut souvent regretter que les systèmes se régulent principalement par les abandons qui ne coïncident pas forcément avec des situations d'échec et font donc apparaître de ce point de vue un grand manque d'équité.

\section{Des finalités contradictoires}

Pour prendre un relief bien particulier dans les systèmes en développement de l'Afrique subsaharienne, cette contradiction des finalités plurielles assignées aux examens est cependant une réalité bien connue et identifiable sous des formes diverses dans de nombreux autres cas. C'est une circonstance très générale qui veut que l'examen soit chargé, pour l'individu, de certifier les acquisitions scolaires qu'il a réalisées en amont, et pour la cohorte, de commander le passage vers l'aval. Il est assez rare qu'un examen n'ait que l'un des deux rôles. Ce fut le cas, en France, du certificat d'études primaires, distinct du passage en sixième ou en primaire supérieur, avant d'ailleurs de disparaître, peut-être de ce fait. Mais dans le même pays, il n'est pas rare d'entendre des universitaires, inquiets de l'embouteillage que provoquent dans les premiers cycles supérieurs des candidats au profil modeste et aux ambitions incertaines, déplorer que le baccalauréat continue d'être le sésame des facultés.

Posons que cette contradiction est au principe même de l'examen et qu'elle est visible ou non selon le profil particulier de la démographie scolaire qu'il a le pouvoir de modeler. Si, à l'endroit de la pyramide où se situe notre examen, naissent une ou plusieurs bonnes raisons d'opérer une régulation malthusienne, alors l'école a mal à son examen. Dans de nombreux cas, le système pourra s'accommoder d'une divergence entre deux finalités, qui ne va pas jusqu'à la contradiction statistique, dans d'autres, comme les cas africains qui nous occupent, la divergence tend vers l'antagonisme et il n'est plus possible d'être à peu près juste tout en gérant les flux de façon à peu près convenable.

\section{QUELLES SOLUTIONS?}

Ce problème appartient à la catégorie de ceux que l'on résout difficilement. Comme c'est fréquemment le cas en pédagogie ou dans la gestion des systèmes éducatifs, il résulte d'une tension à laquelle le système doit s'accommoder.

Les autorités éducatives mauritaniennes ont cependant, en élaborant un mécanisme de certification nouveau et original, opté pour une approche 
radicale de la difficulté, qui correspond au caractère très aigu qu'elle a dans ce pays, tandis que la combinaison d'outils plus classiques permet d'imaginer, dans d'autres cas, des arrangements acceptables.

\section{La solution mauritanienne}

Depuis cinq ans, le système éducatif mauritanien est entré dans une phase de réformes profondes. Unification linguistique, efforts importants pour atteindre des taux d'achèvement convenables pour la scolarité primaire, aggiornamento des méthodes de gestion, maîtrise des flux dans les échelons élevés des scolarités et l'enseignement supérieur. Pour ce dernier point, le plan national de développement du système éducatif est même allé jusqu'à contenir un engagement des autorités à diminuer en valeur absolue le nombre des entrées au lycée, en faisant porter l'essentiel des efforts sur le développement des scolarités primaires et de collège. Les faibles résultats de ce dernier ont par ailleurs amené les autorités à décider de la création d'une quatrième année d'études en collège, au lieu des trois que le pays avait toujours connues, amenant ainsi la scolarité de base à un ensemble de dix années. L'insertion d'une année nouvelle au milieu d'un cursus n'est pas sans poser de redoutables problèmes aux gestionnaires, mais elle a dans notre cas été aussi porteuse d'un atout essentiel pour la gestion des examens, victime de la tension suivante sur les flux : pour cinq enfants qui entrent au collège, un seulement au maximum pourra poursuivre au lycée.

Le dispositif retenu est le suivant. L'examen traditionnel du brevet, en fin de troisième année de collège, est maintenu à ce stade; son rôle est de certifier les acquis scolaires des enfants. On attend de cet examen qu'il valide sans parcimonie des études menées avec profit jusqu'à ce stade. La quatrième année nouvellement créée l'est avec un profil un peu particulier. Il s'agit en fait d'une année préparatoire au lycée, accessible à tous les titulaires du brevet mais qu'il est exclu de redoubler (de façon à éviter la création d'une file d'attente en fin de cycle), et s'achevant sur un concours d'accès au lycée. Ce concours, création nouvelle, a pour mandat de vérifier non des acquis mais des aptitudes à des études longues, les baccalauréats (principalement classiques en Mauritanie) ayant pour vocation principale la poursuite d'études dans l'enseignement supérieur. La vocation principale et affichée du concours est bien la gestion des flux et l'on en attend des résultats tout à fait sélectifs pendant toute la (malheureusement longue) période où la plus grande part des ressources du pays consacrées à l'éducation devront être affectées au développement et à la généralisation des scolarités de base.

La solution trouvée en Mauritanie présente un caractère de rationalité très affirmé ainsi qu'un caractère de publicité tout à fait nouveau. En effet, il est clairement dit aux jeunes qu'on attend d'eux qu'ils poursuivent sans entrave leur scolarité jusqu'au terme du collège, vers l'âge de leurs seize ans environ, mais que le pays ne pourra promouvoir qu'une petite minorité d'entre eux vers 
des études ultérieures : la réussite de la scolarité de base, même poussée à son achèvement, n'est pas donnée comme un gage d'accès au niveau supérieur.

\section{Autres pistes}

À défaut d'une solution aussi radicale (qui n'est pas sans coût et sans difficultés d'explications), des voies acceptables permettant de desserrer les tensions qui pèsent sur l'examen de fin d'école moyenne peuvent être recherchées par la combinaison de mesures déjà connues ou de diverses modalités de certification.

En premier lieu, la possibilité de découpler la certification de fin de cycle d'une année scolaire particulière existe et peut se montrer prometteuse. Par exemple, l'examen serait accessible indifféremment à la fin de chacune des deux dernières années de collège et de la première année de lycée. Cette disposition, rendue possible si les programmes scolaires connaissent une progression concentrique, a le grand mérite de délier la promotion dans les échelons supérieurs et la réussite à l'examen. Ce mérite s'associe à une sorte de "droit à la lenteur» consenti aux élèves les moins brillants. En revanche, cela ne résout pas la gestion des flux vers l'aval, pour laquelle l'administration scolaire n'a le support que de sa propre autorité et des notes obtenues en cours d'année, de sorte que seule une administration scolaire solide et incontestée sera à même de pratiquer sur cette base la nécessaire gestion limitée des entrées au lycée. En revanche, si l'administration scolaire n'est pas en mesure, pour diverses raisons, de garantir de façon crédible un minimum de transparence et d'harmonisation des pratiques des différents établissements, on peut redouter ce type de solution.

On peut également remettre au goût du jour la pratique éprouvée (elle l'est généralement pour la transition primaire-premier cycle secondaire) de l'«examen-concours", dans laquelle un jeu unique d'épreuves sert à certifier l'amont et à commander l'accès à l'aval. Cette solution a pour elle la légèreté du coût mais on sait qu'elle n'a jamais permis d'éviter la constitution d'embouteillages de fin de cycle constitués en grande partie par des enfants qui, reçus à l'examen, redoublent pour l'obtention du concours, en organisant ainsi un certain gaspillage de ressources et en érodant le barrage disposé pour gérer les flux. Sur le plan pédagogique, la pratique de l'examen-concours encourt également le reproche de tenir pour identiques les compétences acquises au cycle d'amont et celles qui seront nécessaires au cycle d'aval, alors que tout porte à croire qu'elles diffèrent sensiblement.

Le contrôle continu peut également être appelé à la rescousse - par exemple pour constituer tout ou partie de l'évaluation sommative de fin de cycle, avec les avantages de coût et d'organisation qui lui sont associés. Il faut cependant que la population l'accepte comme pouvant fonder un diplôme. Certaines sociétés y sont habituées, d'autres non. Le contrôle continu est par 
ailleurs plus facilement la source de dérapages regrettables que les examens organisés de façon plus centralisée ${ }^{2}$, avec entre autres des garanties d'anonymat.

Dans tous les cas de figure, l'examen de fin d'école moyenne dans les systèmes en développement d'Afrique subsaharienne est, et sera pour longtemps, le lieu d'une certaine forme de brutalité. Gageons d'ailleurs que ceux de nos lecteurs qui ne sont pas familiers avec ce contexte auront été à quelque moment choqués par nos affirmations - toutes déduites qu'elles soient de l'observation.

Cela implique que les autorités scolaires ont et auront la tâche non seulement d'élaborer une solution acceptable, compte tenu de leur contexte national propre, mais également d'en expliquer les motifs. Les ressources sont rares et tant que tous les enfants n'auront pas la possibilité effective de terminer le cycle primaire, toute affectation de richesse à un cycle supérieur constitue un début d'injustice. Tout l'horizon visible des systèmes subsahariens est habité par ce simple constat.

Cela implique aussi, naturellement, que l'ensemble pédagogique que constitue l'école moyenne soit conçu comme un cycle terminal et non comme une propédeutique à des études plus poussées : la gestion de l'examen, son contenu, son déroulement, ne peuvent être pensés en dehors de ces considérations pédagogiques premières qui interrogent les finalités de l'école et son rôle au sein de la société.

\section{BIBLIOGRAPHIE}

BERNARD J.-M. et ROBERT F. (2004): Nouveaux enjeux pour l'école moyenne en Afrique, sous presse, Louvain, De Boeck.

COWELL R.N. et HOLSINGER D.B. (2000): Positioning secondary school education in developing countries, IIEP/UNESCO.

GURGAND M. (1993): Éducation et productivité agricole en Côte d'Ivoire, Revue d'économie du développement, $\mathrm{n}^{\circ} 4$.

GURGAND M.: Sait-on mesurer le rôle économique de l'éducation? Une confrontation des résultats empiriques micro et macroéconomiques, texte d'appui au séminaire interne de l'IREDU du 15 avril 2002, en ligne sur le site de l'IREDU, Université de Bourgogne.

KELLAGHAN T., GREANEY V. (2003): Monitoring performance : assessment and examination in Africa, ADEA.

MINGAT A. et SUCHAUT B. (2000): Les systèmes éducatifs africains, une analyse comparative, De Boeck Université.

2. Kellaghan (T.), Greaney (V.), Monitoring performance : assessment and examination in Africa, ADEA, 2003. 
MINGAT A. (2003): Combien d'années de scolarisation pour assurer la rétention de l'alphabétisation dans les pays d'Afrique sub-saharienne? PSAST/AFTHD, Banque mondiale.

MORISSON C. (2002): Santé, éducation et réduction de la pauvreté, Cahiers de politique économique $\mathrm{n}^{\circ} 19$, OCDE.

PRITCHETT L. (1997): Where has all education gone?, working paper 1581, The World Bank.

ROBERT F. (2003): L'examen de fin d'école moyenne en Afrique francophone subsaharienne: nouvelles contraintes, nouveaux enjeux. Actes du $16^{\mathrm{e}}$ colloque international de l'ADMEE-Europe.

Contraintes et espaces de liberté pour le développement en quantité et en qualité de l'éducation au Niger, ministère de l'Éducation nigérien / Banque mondiale, 2000.

Programme décennal de développement de l'éducation au Niger (PDDE 20022012), Diagnostic, orientations, objectifs et stratégies, République du Niger, ministère de l'Éducation, Niamey, juillet 2001.

Le système éducatif togolais, éléments d'analyse pour une revitalisation, ministère de l'Éducation togolais - Banque mondiale, 2002.

Éléments d'appréciation de la qualité de l'enseignement primaire en Afrique francophone, Programme d'analyse des systèmes éducatifs de la CONFEMEN, Biennale de l'ADEA, 2003.

Éducation et formation à Madagascar : vers une politique nouvelle pour la croissance et la réduction de la pauvreté, Banque mondiale, 2001.

Programme national pour l'amélioration de l'enseignement (PNAE2), Ministère de l'enseignement secondaire et de l'éducation de base, Madagascar, 1997.

Plan national de développement du secteur éducatif, Ministère de l'éducation nationale, Nouakchott, 2001.

Programme décennal de l'éducation et de la formation, Ministère de l'éducation nationale, Dakar, 1999.

Déclaration mondiale sur l'éducation pour tous, et cadre d'action pour répondre aux besoins éducatifs fondamentaux, Jomtien, UNESCO, 1990.

L'éducation pour tous : tenir nos engagements collectifs, avec les six cadres d'action régionaux (déclaration du Forum mondial sur l'éducation pour tous, Dakar, avril 2000), UNESCO, 2000.

Recueil de données mondiales sur l'éducation 2003, ISU/UNESCO, Montréal, 2003. 